\title{
Interference effects in heavy Higgs production via gluon fusion in the singlet extension of the Standard Model
}

\section{Ezio Maina}

\author{
INFN, Sezione di Torino, \\ Via Giuria 1, 10125 Torino, Italy \\ Dipartimento di Fisica, Università di Torino, \\ Via Giuria 1, 10125 Torino, Italy \\ E-mail: maina@to.infn.it
}

ABSTRACT: The measurements of the properties of the Higgs boson still leave room for a non minimal scalar sector. Extensions of the Standard Model typically involve multiple neutral Higgs fields which can interfere among themselves. We show that these interference effects can be substantial taking as example the one Higgs Singlet Model, the simplest renormalizable addition to the SM.

KeYwords: Hadronic Colliders

ArXiv EPrint: 1501.02139 


\section{Contents}

1 Introduction 1

2 The Singlet Extension of the Standard Model 1

3 Limits on the parameters 4

4 Interference effect and simulation tools 4

5 Results 5

$\begin{array}{lll}6 & \text { Conclusions } & 10\end{array}$

\section{Introduction}

All mesurements performed in LHC Run I relative to the resonance discovered at about $125 \mathrm{GeV}[1,2]$ are consistent with the hypothesis that the new particle is indeed the Standard Model Higgs boson. While the mass is already known to an astonishing three per mill from the latest published CMS measurement [3], the signal strengths $\mu^{i}=\sigma^{i} / \sigma_{\mathrm{SM}}^{i}$, where $i$ runs over the decay channnels, are known to about 10 to $20 \%$ [3-5]. This leaves room for modifications of the SM with a more complicated Higgs sector provided they are consistent within experimental errors with the data. A larger Higgs sector implies that additional scalar states are present in the spectrum. Direct searches have provided limits on the existence of new spin zero particles and on the strengths of their couplings [6]. The larger luminosity and energy in Run II will allow more precise measurements of the already discovered Higgs properties and extend the mass range in which other scalars can be searched for.

The simplest renormalizable extension of the SM is the one Higgs Singlet Model (1HSM). It introduces one additional real scalar field which is a singlet under all SM gauge groups. The 1HSM has been extensively investigated in the literature [7-31], however, to our knowledge, no public MC implementation of the model is available. In this note we present such an implementation using FeynRules for the derivation of the vertices and Madgraph 5 for the generation of the amplitudes. We then discuss the simple case of Higgs production via gluon fusion at the LHC.

\section{The Singlet Extension of the Standard Model}

The singlet extension of the SM is defined by adding to the standard Lagrangian the following gauge invariant, renormalizable term:

$$
\mathscr{L}_{s}=\partial^{\mu} S \partial_{\mu} S-\mu_{1}^{2} \Phi^{\dagger} \Phi-\mu_{2}^{2} S^{2}+\lambda_{1}\left(\Phi^{\dagger} \Phi\right)^{2}+\lambda_{2} S^{4}+\lambda_{3} \Phi^{\dagger} \Phi S^{2} .
$$


where $S$ is a real $\mathrm{SU}(2)_{L} \otimes \mathrm{U}(1)_{Y}$ singlet and $\Phi$ is the SM Higgs weak isospin doublet. Here and in the following we adopt the notation of ref. [25]. A $\mathcal{Z}_{2}$ symmetry which forbids additional terms in the potential is assumed. A detailed discussion of the 1HSM without $\mathcal{Z}_{2}$ symmetry can be found in refs. [9, 11, 24, 28, 29].

The neutral components of the scalar fields can be expanded around their respective Vacuum Expectation Values (VEVs) as follows:

$$
\Phi=\left(\begin{array}{c}
G^{ \pm} \\
\frac{v_{d}+l^{0}+i G^{0}}{\sqrt{2}}
\end{array}\right) \quad S=\frac{v_{s}+s^{0}}{\sqrt{2}} .
$$

The minimum of the potential is achieved for

$$
\mu_{1}^{2}=\lambda_{1} v_{d}^{2}+\frac{\lambda_{3} v_{s}^{2}}{2} ; \quad \mu_{2}^{2}=\lambda_{2} v_{s}^{2}+\frac{\lambda_{3} v_{d}^{2}}{2}
$$

provided

$$
\lambda_{1}, \lambda_{2}>0 ; \quad 4 \lambda_{1} \lambda_{2}-\lambda_{3}^{2}>0
$$

The mass matrix in the gauge basis can be diagonalized into the (tree-level) mass basis introducing new fields:

$$
h=l^{0} \cos \alpha-s^{0} \sin \alpha \quad \text { and } \quad H=l^{0} \sin \alpha+s^{0} \cos \alpha
$$

with $-\frac{\pi}{2}<\alpha<\frac{\pi}{2}$. The masses are

$$
m_{h, H}^{2}=\lambda_{1} v_{d}^{2}+\lambda_{2} v_{s}^{2} \mp\left|\lambda_{1} v_{d}^{2}-\lambda_{2} v_{s}^{2}\right| \sqrt{1+\tan ^{2}(2 \alpha)}, \quad \tan (2 \alpha)=\frac{\lambda_{3} v_{d} v_{s}}{\lambda_{1} v_{d}^{2}-\lambda_{2} v_{s}^{2}}
$$

with the convention $m_{H}^{2}>m_{h}^{2}$. They correspond to a light $[h]$ and a heavy $[H] \mathcal{C P}$-even mass-eigenstate.

The Higgs sector in this model is determined by five independent parameters, which can be chosen as

$$
m_{h}, m_{H}, \sin \alpha, v_{d}, \tan \beta \equiv v_{d} / v_{s},
$$

where the doublet VEV is fixed in terms of the Fermi constant through $v_{d}^{2}=G_{F}^{-1} / \sqrt{2}$. Furthermore one of the Higgs masses is determined by the LHC measurement of $125.02 \mathrm{GeV}$. Therefore, three parameters of the model are presently free.

It should be mentioned that allowing a discrete symmetry to be spontaneously broken, as is the case in the simplified model considered here when the singlet field $S$ has a non zero vacuum expectation value, will introduce potentially problematic cosmic domain walls [13, 32-36]. These considerations, however have little bearing on the paper's main point as explained in section 4 and will not be discussed further.

As only the doublet component, before mixing, can couple to the fermions (via ordinary Yukawa interactions) and the gauge bosons (via the gauge covariant derivative), all of the 
$\mathrm{H} \rightarrow$ hh Width

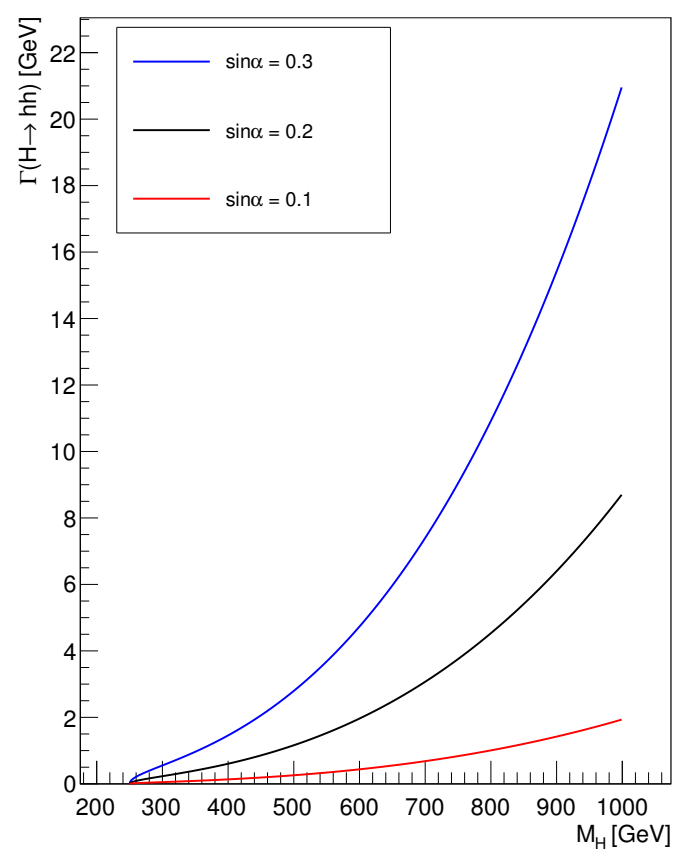

$\mathrm{BR}(\mathrm{H} \rightarrow \mathrm{hh})$

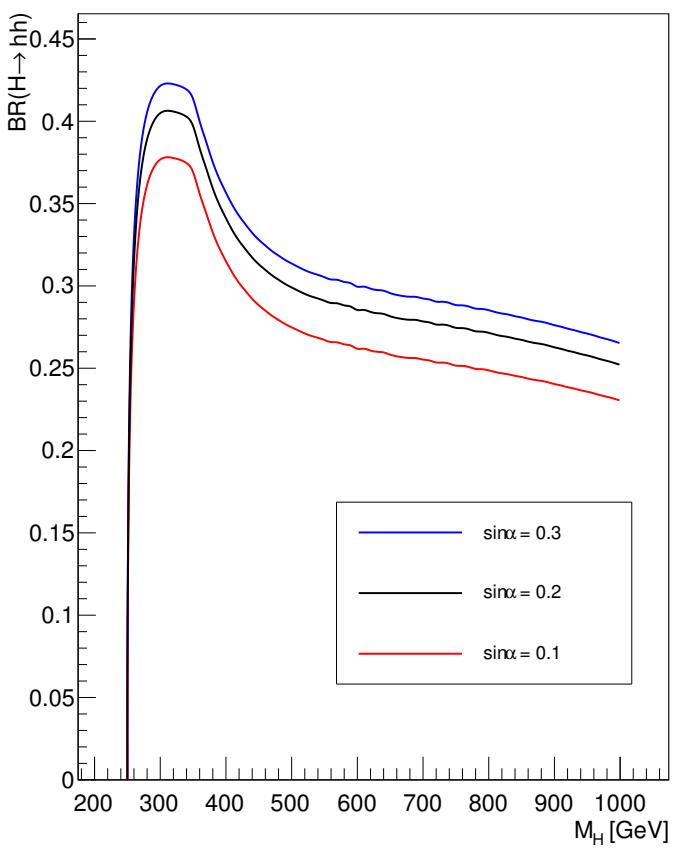

Figure 1. On the left: the partial width for the $H \rightarrow h h$ process for $s_{\alpha}=0.3,0.2,0.1$ and $\tan \beta=1.0$. On the right: the corresponding branching ratios.

corresponding Higgs couplings are rescaled universally, yielding

$$
\begin{aligned}
g_{x x s} & =g_{x x h}^{\mathrm{SM}}\left(1+\Delta_{x s}\right) \quad \text { with } \quad 1+\Delta_{x s}=\left\{\begin{array}{cc}
\cos \alpha & s=h \\
\sin \alpha & s=H
\end{array} .\right. \\
g_{x x s_{1} s_{2}} & =g_{x x h h}^{\mathrm{SM}}\left(1+\Delta_{x s_{1}}\right)\left(1+\Delta_{x s_{2}}\right) .
\end{aligned}
$$

where $x x$ represents a pair of SM fermions or vectors.

The couplings in the scalar sector involve $\tan \beta$ and are slightly more complicated. As an example we reproduce the triple scalar vertices in terms of the independent parameters mentioned above $\left(s_{\alpha}=\sin \alpha, c_{\alpha}=\cos \alpha\right)$ :

$$
\begin{aligned}
V_{h h h} & -\frac{3 i e M_{h}^{2}}{2 M_{W} s_{W}}\left\{c_{\alpha}^{3}-s_{\alpha}^{3} \tan \beta\right\} \\
V_{h H H} & -\frac{i e s_{\alpha} c_{\alpha}}{2 M_{W} s_{W}}\left\{s_{\alpha}-c_{\alpha} \tan \beta\right\}\left\{M_{h}^{2}+2 M_{H}^{2}\right\} \\
V_{H h h} & \left.-\frac{i e s_{\alpha} c_{\alpha}}{2 M_{W} s_{W}}\left\{c_{\alpha}+s_{\alpha} \tan \beta\right\}\right\}\left\{2 M_{h}^{2}+M_{H}^{2}\right\} \\
V_{H H H} & -\frac{3 i e M_{H}^{2}}{2 M_{W} s_{W}}\left\{s_{\alpha}^{3}+c_{\alpha}^{3} \tan \beta\right\}
\end{aligned}
$$

The tree level partial width for the decay of the heavy scalar into two light ones reads:

$$
\Gamma(H \rightarrow h h)=\frac{e^{2} M_{H}^{3}}{128 \pi M_{W}^{2} s_{W}^{2}}\left(1-\frac{4 M_{h}^{2}}{M_{H}^{2}}\right)^{\frac{1}{2}}\left(1+\frac{2 M_{h}^{2}}{M_{H}^{2}}\right)^{2} s_{\alpha}^{2} c_{\alpha}^{2}\left(c_{\alpha}+s_{\alpha} \tan \beta\right)^{2}
$$


In figure 1 we show the partial width and the corresponding branching ratios as a function of the heavy Higgs mass for $s_{\alpha}=0.3,0.2,0.1$ and $\tan \beta=1.0$. The BR is computed as the ratio of the lowest order width in eq. (2.14) to the total width given in ref. [37] multiplied by $s_{\alpha}^{2}$. The BR rises sharply above the kinematical threshold and, for the parameter range we have considered, remains larger than $25 \%$ up to $M_{H}=1 \mathrm{TeV}$. This raises the tantalizing prospect of a relatively abundant production of heavy Higgses followed by their decay into two light ones if $M_{H}>2 M_{h}$. See ref. [29] for a detailed discussion in the framework of the 1SHM.

\section{Limits on the parameters}

The strongest limits on the parameters of the 1HSM come from measurements of the coupling strengths of the light Higgs [3-6], which dominate for small masses of the heavy Higgs, and from the contribution of higher order corrections to precision measurements, in particular to the mass of the $\mathrm{W}$ boson [26], which provides the tightest constraint for large $M_{H}$. The most precise result for the overall coupling strength of the Higgs boson from CMS [3] reads

$$
\hat{\mu}=\hat{\sigma} / \sigma_{\mathrm{SM}}=1.00 \pm 0.13
$$

Therefore the absolute value of $\sin \alpha$ cannot be larger than about 0.4. This is in agreement with the limits obtained in refs. $[26,30]$ which conclude that the largest possible value for the absolute value of $\sin \alpha$ is 0.46 for $M_{H}$ between 160 and $180 \mathrm{GeV}$. This limit becomes slowly more stringent for increasing heavy Higgs masses reaching about 0.2 at $M_{H}=700 \mathrm{GeV}$.

\section{Interference effect and simulation tools}

The focal point of this note is the interference between the two Higgs fields. In general, any amplitude involving a single Higgs exchange can be written as

$$
A=A^{\prime}\left(\frac{c_{\alpha}^{2}}{q^{2}-M_{h}^{2}+i \Gamma_{h} M_{h}}+\frac{s_{\alpha}^{2}}{q^{2}-M_{H}^{2}+i \Gamma_{H} M_{H}}\right)+A_{0}=A_{1}+A_{0}
$$

where $A_{0}$ does not involve the scalar fields. The real parts of the two propagators intefere destructively for $M_{h}^{2}<q^{2}<M_{H}^{2}$ and constructively for $q^{2}<M_{h}^{2}$ and $M_{H}^{2}<q^{2}$. This phenomenon has already been noticed in ref. [27] where however it was dismissed as numerically irrelevant.

As will be argued in section 5 , the interference effects can be substantial, their relevance increasing with $M_{H}$. The shape of the the heavier Higgs peak is also strongly affected and this will need to be taken into account in any search for additional scalars and eventually in the measurement of their properties.

These features are neglected by any prediction based on the narrow width approximation, or equivalently on a production times decay approach.

Clearly, this interference between different Higgs fields is not a peculiarity of the Singlet Model. It will indeed occur in any theory with multiple scalars which couple to the same set of elementary particles, albeit possibly with different strengths. For instance, in the 
MSSM the couplings of the two $\mathcal{C P}$-even Higgs fields to vector bosons and fermions are equal to the SM ones multiplied by simple trigonometric functions of the mixing angle $\alpha$ and of $\beta$, the arctangent of the ratio of the vacuum expectation values. As a consequence, effects similar to those discussed in this paper are to be expected in the MSSM.

The relevance of the interference term does depend on the relative size of $A_{0}$ and $A_{1}$ in eq. (4.1) and in particular on the couplings of the scalars to the rest of the spectrum which in general will not follow the simple pattern which characterizes the 1HSM. The effect is expected to be significant for processes in which the resonant part of the amplitude is large as in gluon fusion for large values of the heavy Higgs mass.

In order to allow for the Monte Carlo simulation of the 1HSM we have used FeynRules [38, 39] to prepare a UFO file [40] for the model, which can be imported, as we did, in MadGraph 5 [41] and many other general purpose MC tools. It enables the simulation at tree level of any process in the 1HSM. The UFO file can be downloaded from http://personalpages.to.infn.it/ maina/Singlet.

The gluon fusion channel in MadGraph requires particular care. MadGraph treats the gluon-Higgs effective vertex in the narrow width approximation, through an expansion of the top loop amplitude in powers of $M_{h}^{2} / M_{\text {top }}^{2}$, which is unsuitable in the present context and which fails for Higgs masses above the t-tbar threshold. The appropriate effective vertex $V_{g g h}$ must be introduced by hand in the matrix element:

$$
V_{g g h}=-i \frac{2 \alpha_{s}\left(\sqrt{2} G_{F}\right)^{\frac{1}{2}} M_{\mathrm{top}}^{2}}{\pi \hat{s}}\left(1-\frac{1}{2}(1-\tau) C(\tau)\right),
$$

with

$$
C(\tau)= \begin{cases}-2 \arcsin (1 / \sqrt{\tau})^{2} & \tau>1 \\ \frac{1}{2}\left(\log \left(\frac{1+\beta}{1-\beta}\right)-i \pi\right)^{2} & \tau<1\end{cases}
$$

where $\tau=4 M_{\text {top }}^{2} / \hat{s}, \beta=\sqrt{1-\tau}$ and $\hat{s}$ is the square of the sum of the momenta carried by the gluons which in general is not equal to the Higgs mass squared.

The tensor structure of the vertex is already taken care of by MadGraph.

\section{Results}

As an example we have studied Higgs production through gluon fusion in the four electron channel, $g g \rightarrow h, H \rightarrow Z Z \rightarrow 4 e$, at the LHC with a center of mass energy of $13 \mathrm{TeV}$.

We neglect the non resonant contribution given by $q \bar{q} \rightarrow Z Z$ and by $g g \rightarrow Z Z$ through a quark box amplitude. This continuum is known to be large and in particular there is a non negligible interference between the box contribution and Higgs production through the heavy quark three point loop. These terms are well known [42-55] and are essential for accurate phenomenological predictions. The region of large invariant masses of the four final state leptons in $g g \rightarrow Z Z, W W \rightarrow 4 l$ has been studied in detail in ref. [55] in the SM with a Higgs of $125 \mathrm{GeV}$. Above the light Higgs peak, the differential cross 


\section{4 invariant mass}

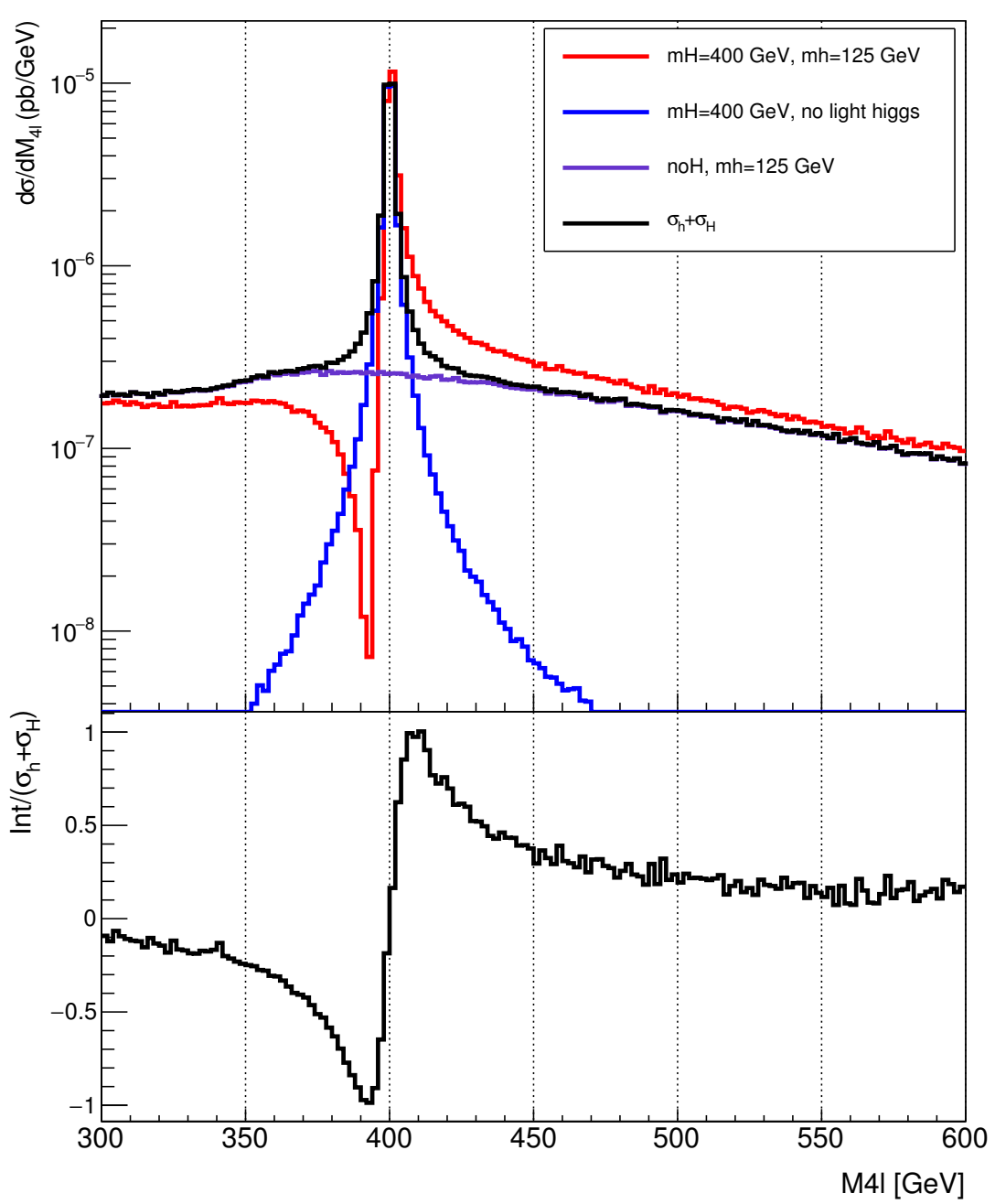

Figure 2. $g g \rightarrow h, H \rightarrow Z Z \rightarrow 4 e$ at the LHC with a center of mass energy of $13 \mathrm{TeV}$. Upper part:four lepton invariant mass distribution for $M_{h}=125 \mathrm{GeV}, M_{H}=400 \mathrm{GeV}$ and $s_{\alpha}=0.2$. The red line is the full result $(\sigma)$. The violet histograms $\left(\sigma_{h}\right)$ shows the SM prediction with Higgs couplings scaled by $\cos \alpha$. The blue line $\left(\sigma_{H}\right)$ gives the result when the light Higgs diagrams are neglected while the $H \rightarrow h h$ contribution to $\Gamma_{H}$ is retained. The black histogram $\left(\sigma_{N I}\right)$ refers to the sum of the violet and blue lines and corresponds to neglecting the interference effects. Lower part: the ratio $\frac{\sigma-\sigma_{N I}}{\sigma_{N I}}$.

section is dominated by the continuum for $M_{4 l}<2 M_{\text {top }}$. The box contribution however drops more rapidly with increasing $M_{4 l}$ than the Higgs mediated one. Unitarity requires the interference between these two components to be negative and while it is essentially negligible below the top threshold it becomes more relevant for larger masses and exceeds $50 \%$ of the incoherent sum of the two contributions in the one $\mathrm{TeV}$ range.

Our main results are shown in figures $2-5$ and table 1 . No cut has been applied to the final state. We have used CTEQ6L1 parton distribution functions [56]. The ratio of vacuum expectation values $\tan \beta$ has been taken equal to one. 


\section{4 invariant mass}

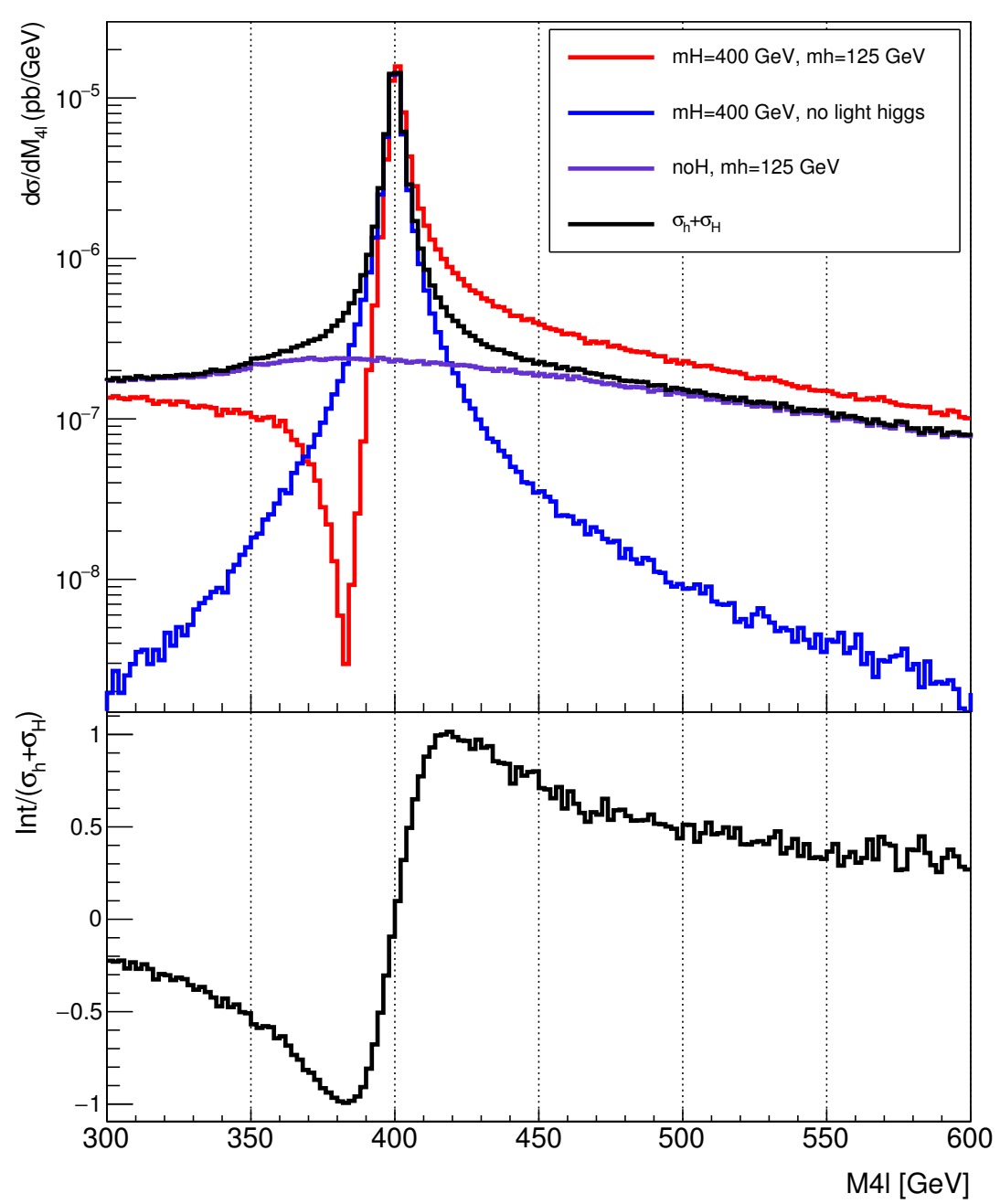

Figure 3. Four lepton invariant mass distribution for $M_{h}=125 \mathrm{GeV}, M_{H}=400 \mathrm{GeV}, s_{\alpha}=0.3$. The meaning of the various histograms is as in figure 2.

The width of the light and heavy scalar are

$$
\Gamma_{h}=\Gamma^{\mathrm{SM}}\left(M_{h}\right) c_{\alpha}^{2}, \quad \Gamma_{H}=\Gamma^{\mathrm{SM}}\left(M_{H}\right) s_{\alpha}^{2}+\Gamma(H \rightarrow h h)
$$

This corresponds, using eq. (2.14) for the $H \rightarrow h h$ width and ref. [37] for the SM Higgs width, to $\Gamma_{H}=1.77(4.08) \mathrm{GeV}$ for $M_{H}=400 \mathrm{GeV}, s_{\alpha}=0.2(0.3) ; \Gamma_{H}=15.80 \mathrm{GeV}$ for $M_{H}=600 \mathrm{GeV}$ and $s_{\alpha}=0.3 ; \Gamma_{H}=16.69 \mathrm{GeV}$ for $M_{H}=800 \mathrm{GeV}$ and $s_{\alpha}=0.2$. We have assumed $M_{h}=125 \mathrm{GeV}$ which corresponds to $\Gamma^{\mathrm{SM}}(125)=4.03 \mathrm{MeV}$.

We show the region around the heavy Higgs peak where the interference affects are most prominent. The invariant mass distribution in the neighborhood of the light Higgs resonance is unaffected within the accuracy of the present simulation. In all figures the violet histogram is the result obtained including only the light Higgs with SM couplings scaled by $c_{\alpha}$ which we denote as $\sigma_{h}$. Since $\Gamma_{h}$ is small $\sigma_{h} \approx \sigma_{\mathrm{SM}}\left(M_{h}\right) c_{\alpha}^{4}$ for large $M_{4 l}$. 


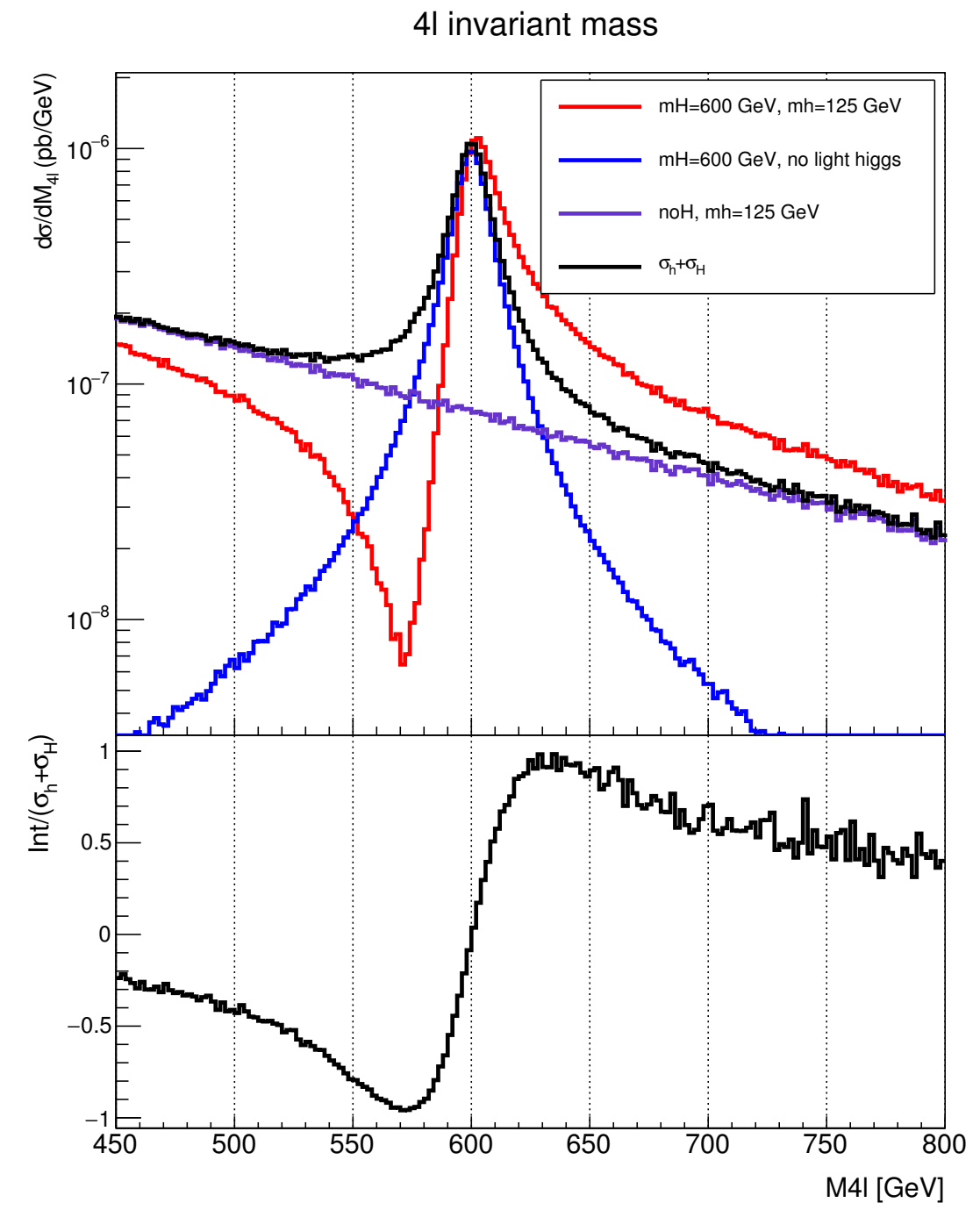

Figure 4. Four lepton invariant mass distribution for $M_{h}=125 \mathrm{GeV}, M_{H}=600 \mathrm{GeV}, s_{\alpha}=0.3$. The meaning of the various histograms is as in figure 2 .

\begin{tabular}{|c|c|c|c|c|c|c|}
\hline & \multicolumn{3}{|c|}{$200 \mathrm{GeV}<M_{4 l}<1 \mathrm{TeV}$} & \multicolumn{3}{c|}{$M_{H}-25 \mathrm{GeV}<M_{4 l}<M_{H}+25 \mathrm{GeV}$} \\
\hline & $\sigma$ & $\sigma_{h}+\sigma_{H}$ & $\sigma_{H}$ & $\sigma$ & $\sigma_{h}+\sigma_{H}$ & $\sigma_{H}$ \\
\hline$M_{H}=400 \mathrm{GeV}, s_{\alpha}=0.2$ & 72.95 & 70.96 & 26.00 & 32.09 & 32.13 & 25.55 \\
\hline$M_{H}=400 \mathrm{GeV}, s_{\alpha}=0.3$ & 101.48 & 96.51 & 55.99 & 60.36 & 59.70 & 53.74 \\
\hline$M_{H}=600 \mathrm{GeV}, s_{\alpha}=0.3$ & 48.44 & 52.52 & 11.99 & 11.51 & 11.96 & 9.97 \\
\hline$M_{H}=800 \mathrm{GeV}, s_{\alpha}=0.2$ & 43.96 & 45.96 & 1.00 & 1.57 & 1.46 & 0.65 \\
\hline
\end{tabular}

Table 1. Cross sections in ab for $g g \rightarrow h, H \rightarrow Z Z \rightarrow 4 e$ at the LHC with a center of mass energy of $13 \mathrm{TeV}$. 


\section{4 invariant mass}

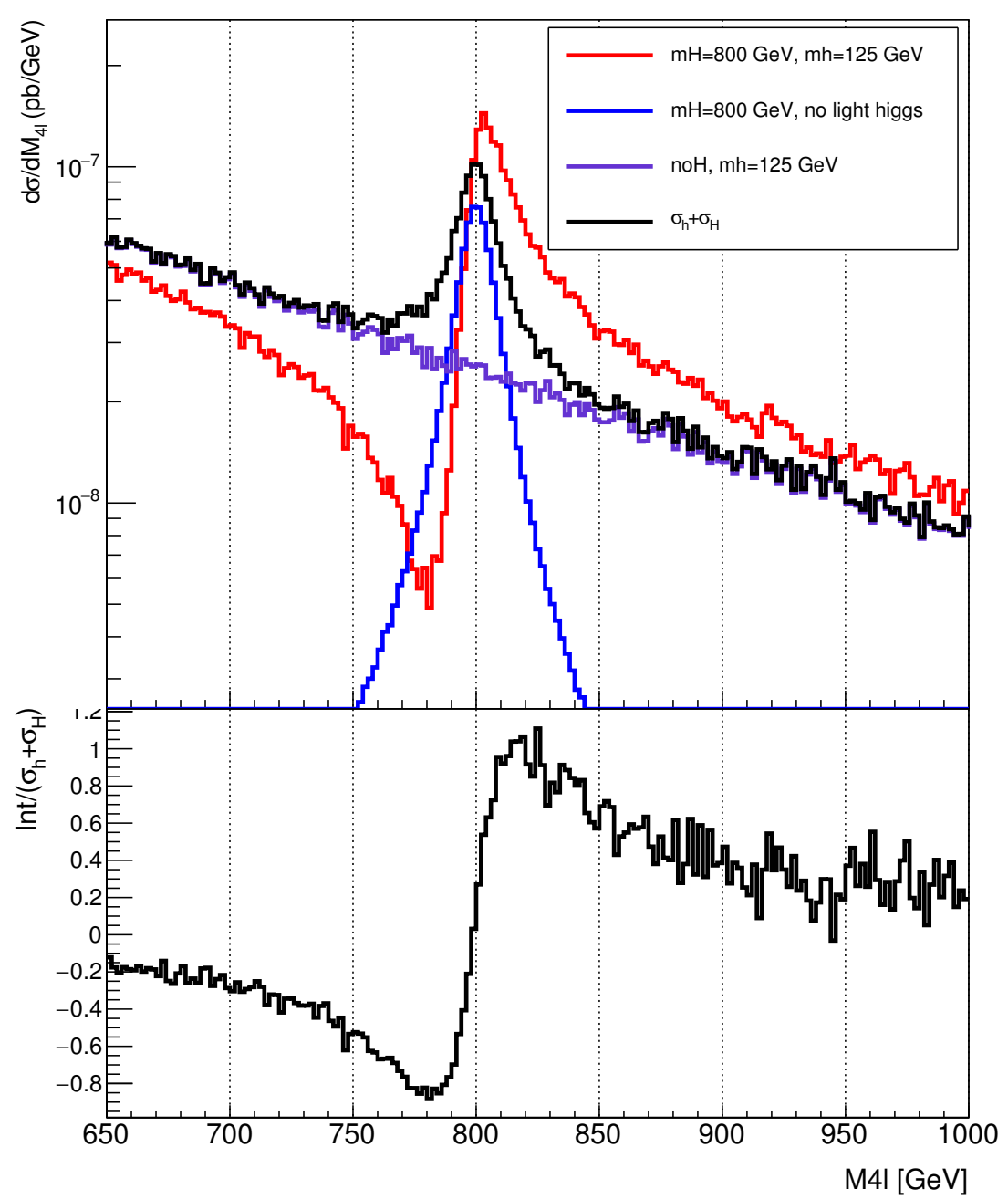

Figure 5. Four lepton invariant mass distribution for $M_{h}=125 \mathrm{GeV}, M_{H}=800 \mathrm{GeV}, s_{\alpha}=0.2$. The meaning of the various histograms is as in figure 2.

The blue line shows the cross section when only the heavy Higgs is present, which will be referred to as $\sigma_{H}$. The $H \rightarrow h h$ contribution to $\Gamma_{H}$ is retained and therefore $\sigma_{H}$ is not equal to $\sigma_{\mathrm{SM}}\left(M_{H}\right) s_{\alpha}^{4}$. The full result, $\sigma$, is shown in red in figure 2 for $s_{\alpha}=0.2, M_{H}=400 \mathrm{GeV}$, in figures 3, 4 for $s_{\alpha}=0.3$ with $M_{H}=400 \mathrm{GeV}$ and $M_{H}=600 \mathrm{GeV}$ respectively and finally in figure 5 for $s_{\alpha}=0.2, M_{H}=800 \mathrm{GeV}$. The black histogram displays the sum of the blue and violet lines, $\sigma_{N I}=\sigma_{h}+\sigma_{H}$, and corresponds to neglecting the interference between the two scalars. The fractional size of the correction to $\sigma_{N I}$ is displayed in the bottom part of the figures where the ratio of the interference term and $\sigma_{N I}, \frac{\sigma-\sigma_{N I}}{\sigma_{N I}}$, is shown.

Contrary to naive expectations the light Higgs contribution is non negligible outside the peak region [55] and the interference effect is substantial. While the details depend obviously on the mass of the heavy Higgs and on the mixing angle, we find a decrease of 10 to $20 \%$ of the differential cross section at invariant masses of the four leptons of $300 \mathrm{GeV}$ for $M_{H}=400$ and of about $20 \%$ for $M_{4 l}=350, M_{H}=600$ and for $M_{4 l}=500$, 
$M_{H}=800$. This depletion becomes more pronounced as $M_{4 l}$ increases and reaches a dip which is almost two orders of magnitude smaller than the predictions which neglect the interplay of the two Higgs fields. The interference stays negative for four lepton masses below the heavy Higgs mass and then turns positive. It attains a maximun in which the true value is about a factor of two larger than $\sigma_{N I}$ and then slowly decreases. The position of the peak is shifted to slightly larger masses. At four lepton masses about $200 \mathrm{GeV}$ larger than $M_{H}$ the interference still amounts to about 20 to $40 \%$ of $\sigma_{N I}$. Because of unitarity, for very large $M_{4 l}$ the full cross section $\sigma$ must approach the SM result $\sigma_{\mathrm{SM}}$. In this region, where the width of the two Higgses can be neglected, $\sigma_{\mathrm{SM}}=\sigma_{h} / c_{\alpha}^{4}=\sigma_{H} / s_{\alpha}^{4}$.

Table 1 shows the cross section in ab for two mass intervals: $200 \mathrm{GeV}<M_{4 l}<1 \mathrm{TeV}$, which roughly coincides with the range employed so far by the experimental collaborations to set limits on the presence and couplings of additional scalars, and $M_{H}-25 \mathrm{GeV}<M_{4 l}<$ $M_{H}+25 \mathrm{GeV}$, as an indication of the possible effects on an analysis in smaller mass bins which requires high luminosity. In the first case, the contribution of the heavy Higgs is a relatively small fraction of the Higgs production cross section in gluon fusion. Furthermore, the interference effects in the $200 \mathrm{GeV}<M_{4 l}<1 \mathrm{TeV}$ depend crucially on the heavy Higgs mass. For $M_{H}=400$ the exact result is larger than the incoherent sum $\sigma_{h}+\sigma_{H}$. The long tail for $M_{4 l}>M_{H}$ gives a larger contribution than the intermediate region $200 \mathrm{GeV}$ $<M_{4 l}<M_{H}$. On the contrary, for larger heavy Higgs masses we have $\sigma<\sigma_{h}+\sigma_{H}$. The negative interference in the intermediate region outweights the positive contribution at larger masses. In the smaller range, $M_{H}-25 \mathrm{GeV}<M_{4 l}<M_{H}+25 \mathrm{GeV}$, the positive and negative contributions are very close for $M_{H}=400 \mathrm{GeV}$ and the full result is in rough agreement with $\sigma_{h}+\sigma_{H}$. For this value of $M_{H}$ and mass interval, the heavy Higgs contribution is significantly larger than the light Higgs one. For $s_{\alpha}=0.3, M_{H}=600 \mathrm{GeV}$ the exact cross section is about $4 \%$ smaller than the incoherent sum while for $s_{\alpha}=0.2$, $M_{H}=800 \mathrm{GeV}$ it is approximately $8 \%$ larger. We see that for large values of the heavy Higgs mass the interference effects are non negligible even on a restricted mass range.

Our results have no pretense to be a complete prediction. They need to be validated with the inclusion of the continuum contribution and of higher order corrections. It should be noticed that, for Higgs decay to color neutral final states, all relevant amplitudes in QCD will have the structure of eq. (4.1) and therefore interference effects between the scalar fields will not be spoiled by QCD corrections.

The interference with the quark box amplitude deserves more care. The gluon-gluon continuum term does not involve scalar exchanges and therefore it cannot be cast in the form of eq. (4.1) and could in principle dilute the effect. However, the interference between the box diagrams and the light Higgs mediated ones is always negative, while the amplitude with a heavy Higgs exchange changes sign at the resonance. As a consequence the continuum and the heavy Higgs term are expected to be in phase for $M_{h}<M_{4 l}<M_{H}$ and out of phase for $M_{4 l}>M_{H}$. A detailed study of this topic is in preparation.

\section{Conclusions}

In any theory with multiple neutral Higgs which couple to the same set of elementary particles the scalars are expected to interfere. We have shown in the case of Higgs produc- 
tion through gluon fusion in the 1SHM that the interference effects can be significant and cannot be neglected when aiming for high accuracy predictions.

\section{Acknowledgments}

Daily discussions with Alessandro Ballestrero have been invaluable and are gratefully acknowledged.

This work has been supported by MIUR (Italy) under contract 2010YJ2NYW_006, by the Compagnia di San Paolo under contract ORTO11TPXK and by the European Union Initial Training Network HiggsTools (PITN-GA-2012-316704).

Open Access. This article is distributed under the terms of the Creative Commons Attribution License (CC-BY 4.0), which permits any use, distribution and reproduction in any medium, provided the original author(s) and source are credited.

\section{References}

[1] ATLAS collaboration, Observation of a new particle in the search for the standard model Higgs boson with the ATLAS detector at the LHC, Phys. Lett. B 716 (2012) 1 [arXiv: 1207.7214] [INSPIRE].

[2] CMS collaboration, Observation of a new boson at a mass of $125 \mathrm{GeV}$ with the CMS experiment at the LHC, Phys. Lett. B 716 (2012) 30 [arXiv:1207.7235] [INSPIRE].

[3] CMS collaboration, Precise determination of the mass of the Higgs boson and tests of compatibility of its couplings with the standard model predictions using proton collisions at 7 and $8 \mathrm{TeV}$, Eur. Phys. J. C 75 (2015) 212 [arXiv:1412.8662] [INSPIRE].

[4] ATLAS collaboration, Updated coupling measurements of the Higgs boson with the ATLAS detector using up to $25 \mathrm{fb}^{-1}$ of proton-proton collision data, ATLAS-CONF-2014-009 (2014).

[5] ATLAS collaboration, Constraints on new phenomena via Higgs coupling measurements with the ATLAS detector, ATLAS-CONF-2014-010 (2014).

[6] CMS collaboration, Searches for new processes in the scalar sector at the CMS experiment, CMS-CR-2014/091 (2014).

[7] V. Silveira and A. Zee, Scalar phantoms, Phys. Lett. B 161 (1985) 136 [inSPIRE].

[8] R. Schabinger and J.D. Wells, A minimal spontaneously broken hidden sector and its impact on Higgs boson physics at the large hadron collider, Phys. Rev. D 72 (2005) 093007 [hep-ph/0509209] [INSPIRE].

[9] D. O'Connell, M.J. Ramsey-Musolf and M.B. Wise, Minimal extension of the standard model scalar sector, Phys. Rev. D 75 (2007) 037701 [hep-ph/0611014] [INSPIRE].

[10] O. Bahat-Treidel, Y. Grossman and Y. Rozen, Hiding the Higgs at the LHC, JHEP 05 (2007) 022 [hep-ph/0611162] [INSPIRE].

[11] V. Barger, P. Langacker, M. McCaskey, M.J. Ramsey-Musolf and G. Shaughnessy, LHC phenomenology of an extended standard model with a real scalar singlet, Phys. Rev. D 77 (2008) 035005 [arXiv:0706.4311] [INSPIRE]. 
[12] G. Bhattacharyya, G.C. Branco and S. Nandi, Universal doublet-singlet Higgs couplings and phenomenology at the CERN Large Hadron Collider, Phys. Rev. D 77 (2008) 117701 [arXiv: 0712.2693] [INSPIRE].

[13] V. Barger, P. Langacker, M. McCaskey, M. Ramsey-Musolf and G. Shaughnessy, Complex singlet extension of the standard model, Phys. Rev. D 79 (2009) 015018 [arXiv:0811.0393] [INSPIRE].

[14] M. Gonderinger, Y. Li, H. Patel and M.J. Ramsey-Musolf, Vacuum stability, perturbativity and scalar singlet dark matter, JHEP 01 (2010) 053 [arXiv:0910.3167] [INSPIRE].

[15] S. Dawson and W. Yan, Hiding the Higgs boson with multiple scalars, Phys. Rev. D 79 (2009) 095002 [arXiv:0904.2005] [INSPIRE].

[16] S. Bock, R. Lafaye, T. Plehn, M. Rauch, D. Zerwas et al., Measuring hidden Higgs and strongly-interacting Higgs scenarios, Phys. Lett. B 694 (2010) 44 [arXiv:1007.2645] [INSPIRE].

[17] P.J. Fox, D. Tucker-Smith and N. Weiner, Higgs friends and counterfeits at hadron colliders, JHEP 06 (2011) 127 [arXiv:1104.5450] [INSPIRE].

[18] C. Englert, T. Plehn, D. Zerwas and P.M. Zerwas, Exploring the Higgs portal, Phys. Lett. B 703 (2011) 298 [arXiv:1106.3097] [INSPIRE].

[19] C. Englert, J. Jaeckel, E. Re and M. Spannowsky, Evasive Higgs maneuvers at the LHC, Phys. Rev. D 85 (2012) 035008 [arXiv:1111.1719] [INSPIRE].

[20] B. Batell, S. Gori and L.-T. Wang, Exploring the Higgs portal with 10/fb at the LHC, JHEP 06 (2012) 172 [arXiv: 1112.5180] [INSPIRE].

[21] C. Englert, T. Plehn, M. Rauch, D. Zerwas and P.M. Zerwas, LHC: standard Higgs and hidden Higgs, Phys. Lett. B 707 (2012) 512 [arXiv:1112.3007] [INSPIRE].

[22] R.S. Gupta and J.D. Wells, Higgs boson search significance deformations due to mixed-in scalars, Phys. Lett. B 710 (2012) 154 [arXiv:1110.0824] [InSPIRE].

[23] B. Batell, D. McKeen and M. Pospelov, Singlet neighbors of the Higgs boson, JHEP 10 (2012) 104 [arXiv: 1207.6252] [INSPIRE].

[24] J.M. No and M. Ramsey-Musolf, Probing the Higgs portal at the LHC through resonant di-Higgs production, Phys. Rev. D 89 (2014) 095031 [arXiv:1310.6035] [INSPIRE].

[25] G.M. Pruna and T. Robens, Higgs singlet extension parameter space in the light of the LHC discovery, Phys. Rev. D 88 (2013) 115012 [arXiv:1303.1150] [INSPIRE].

[26] D. López-Val and T. Robens, $\Delta r$ and the $W$-boson mass in the singlet extension of the standard model, Phys. Rev. D 90 (2014) 114018 [arXiv:1406.1043] [INSPIRE].

[27] C. Englert, Y. Soreq and M. Spannowsky, Off-shell Higgs coupling measurements in BSM scenarios, arXiv:1410.5440 [INSPIRE].

[28] S. Profumo, M.J. Ramsey-Musolf, C.L. Wainwright and P. Winslow, Singlet-catalyzed electroweak phase transitions and precision Higgs boson studies, Phys. Rev. D 91 (2015) 035018 [arXiv: 1407.5342] [INSPIRE].

[29] C.-Y. Chen, S. Dawson and I.M. Lewis, Exploring resonant di-Higgs boson production in the Higgs singlet model, Phys. Rev. D 91 (2015) 035015 [arXiv: 1410.5488] [INSPIRE].

[30] T. Robens and T. Stefaniak, Status of the Higgs singlet extension of the standard model after LHC Run 1, arXiv:1501.0223. 
[31] H.E. Logan, Hiding a Higgs width enhancement from off-shell $g g\left(\rightarrow h^{*}\right) \rightarrow Z Z$ measurements, arXiv:1412.7577 [INSPIRE].

[32] Y. Zeldovich, I.Y. Kobzarev and L.B. Okun, Cosmological consequences of the spontaneous breakdown of discrete symmetry, Zh. Eksp. Teor. Fiz. 67 (1974) 3 [InSPIRE].

[33] T.W.B. Kibble, Topology of cosmic domains and strings, J. Phys. A 9 (1976) 1387 [inSPIRE].

[34] T.W.B. Kibble, Some implications of a cosmological phase transition, Phys. Rept. 67 (1980) 183 [INSPIRE].

[35] S.A. Abel, S. Sarkar and P.L. White, On the cosmological domain wall problem for the minimally extended supersymmetric standard model, Nucl. Phys. B 454 (1995) 663 [hep-ph/9506359] [INSPIRE].

[36] A. Friedland, H. Murayama and M. Perelstein, Domain walls as dark energy, Phys. Rev. D 67 (2003) 043519 [astro-ph/0205520] [INSPIRE].

[37] LHC Higgs Cross Section Working Group collaboration, S. Heinemeyer et al., Handbook of LHC Higgs Cross Sections: 3. Higgs Properties, arXiv:1307.1347 [INSPIRE].

[38] N.D. Christensen and C. Duhr, FeynRules - Feynman rules made easy, Comput. Phys. Commun. 180 (2009) 1614 [arXiv:0806.4194] [INSPIRE].

[39] A. Alloul, N.D. Christensen, C. Degrande, C. Duhr and B. Fuks, FeynRules 2.0 - A complete toolbox for tree-level phenomenology, Comput. Phys. Commun. 185 (2014) 2250 [arXiv:1310.1921] [INSPIRE].

[40] C. Degrande et al., UFO - The Universal FeynRules Output, Comput. Phys. Commun. 183 (2012) 1201 [arXiv: 1108.2040] [INSPIRE].

[41] J. Alwall et al., The automated computation of tree-level and next-to-leading order differential cross sections and their matching to parton shower simulations, JHEP 07 (2014) 079 [arXiv: 1405.0301] [INSPIRE].

[42] E.W.N. Glover and J.J. van der Bij, Vector boson pair production via gluon fusion, Phys. Lett. B 219 (1989) 488 [INSPIRE].

[43] E.W.N. Glover and J.J. van der Bij, Z boson pair production via gluon fusion, Nucl. Phys. B 321 (1989) 561 [INSPIRE].

[44] T. Binoth, M. Ciccolini, N. Kauer and M. Krämer, Gluon-induced W-boson pair production at the LHC, JHEP 12 (2006) 046 [hep-ph/0611170] [INSPIRE].

[45] E. Accomando, The process $g g \rightarrow W W$ as a probe into the EWSB mechanism, Phys. Lett. B 661 (2008) 129 [arXiv:0709.1364] [INSPIRE].

[46] J.M. Campbell, R.K. Ellis and C. Williams, Gluon-gluon contributions to $W^{+} W^{-}$production and Higgs interference effects, JHEP 10 (2011) 005 [arXiv:1107.5569] [INSPIRE].

[47] N. Kauer, Signal-background interference in $g g \rightarrow H \rightarrow V V$, PoS (RADCOR2011) 027 [arXiv: 1201.1667] [INSPIRE].

[48] L.J. Dixon and M.S. Siu, Resonance continuum interference in the diphoton Higgs signal at the LHC, Phys. Rev. Lett. 90 (2003) 252001 [hep-ph/0302233] [INSPIRE].

[49] L.J. Dixon and Y. Sofianatos, Resonance-continuum interference in light Higgs boson production at a photon collider, Phys. Rev. D 79 (2009) 033002 [arXiv:0812.3712] [INSPIRE]. 
[50] E. Accomando et al., Interference effects in heavy $W^{\prime}$-boson searches at the LHC, Phys. Rev. D 85 (2012) 115017 [arXiv:1110.0713] [INSPIRE].

[51] J.M. Campbell, R.K. Ellis and C. Williams, Vector boson pair production at the LHC, JHEP 07 (2011) 018 [arXiv: 1105.0020] [INSPIRE].

[52] R. Frederix et al., Four-lepton production at hadron colliders: aMC@NLO predictions with theoretical uncertainties, JHEP 02 (2012) 099 [arXiv: 1110.4738] [INSPIRE].

[53] T. Melia, K. Melnikov, R. Rontsch, M. Schulze and G. Zanderighi, Gluon fusion contribution to $W^{+} W^{-}+$jet production, JHEP 08 (2012) 115 [arXiv:1205.6987] [INSPIRE].

[54] P. Agrawal and A. Shivaji, Di-vector boson + jet production via gluon fusion at hadron colliders, Phys. Rev. D 86 (2012) 073013 [arXiv:1207.2927] [INSPIRE].

[55] N. Kauer and G. Passarino, Inadequacy of zero-width approximation for a light Higgs boson signal, JHEP 08 (2012) 116 [arXiv:1206.4803] [INSPIRE].

[56] J. Pumplin et al., New generation of parton distributions with uncertainties from global QCD analysis, JHEP 07 (2002) 012 [hep-ph/0201195] [INSPIRE]. 